leee Journal Of Selected Topics In Applied Earth Observations And Remote Sensing

November 2016, Volume 9 Issue 11 Pages 4929-4940

http://dx.doi.org/10.1109/JSTARS.2016.2587840

http://archimer.ifremer.fr/doc/00363/47460/

(c) 2016 IEEE. Personal use is permitted, but republication/redistribution

requires IEEE permission.

\title{
Wave Breaking in Slicks: Impacts on C-Band Quad- Polarized SAR Measurements
}

\author{
Wergeland Morten ${ }^{1}$, Kudryavtsev Vladimir ${ }^{2,3,4}$, Chapron Bertrand ${ }^{5}$, Brekke Camilla ${ }^{6}$, \\ Johannessen Johnny A. ${ }^{1}$
}

\footnotetext{
${ }^{1}$ Nansen Environm \& Remote Sensing Ctr, N-5006 Bergen, Norway.

${ }^{2}$ Russian State Hydrometeorol Univ, St Petersburg 195196, Russia.

${ }^{3}$ Marine Hydrophys Inst, Sevastopol 99011, Ukraine.

${ }^{4}$ Nansen Environm \& Remote Sensing Ctr, N-5006 Bergen, Norway.

${ }^{5}$ Ifremer CERSAT, F-29280 Plouzane, France.

${ }^{6}$ Univ Tromso, Dept Phys \& Technol, N-9019 Tromso, Norway.
}

Corresponding author email addresses : morten.hansen@nersc.no ; kudr@rshu.ru ; bertrand.chapron@ifremer.fr ; camilla.brekke@uit.no ; johnny.johannessen@nersc.no

\begin{abstract}
:
Radarsat-2 C-band quad-polarization SAR observations of crude oil, emulsion, and plant oil slicks acquired in the wind speed range from 4 to $8 \mathrm{~m} / \mathrm{s}$ and incidence angles from 30 degrees to 50 degrees are analyzed to yield new insights into the attenuation of short waves and breaking waves by surface slicks in real conditions. To provide a direct quantitative assessment of the surface wave damping, the measurements are decomposed into polarization difference (PD), polarization ratio (PR), nonpolarized (NP), and cross-polarized (CP) components. The PD signals relate to the extent of Bragg damping in the slick areas, over which the PR is systematically higher than over the ambient sea surface. Attenuation of the breaking waves is revealed to affect both the CP and the NP signals, with distinct but weaker contrasts compared to that of the PD. A revised physical model description is proposed to provide consistent interpretation of the polarized and NP signals. The results suggest that the different slick types and look-alikes can be efficiently discriminated and classified.
\end{abstract}

Keywords : Oil pollution, radar imaging, sea surface, surface waves 


\section{INTRODUCTION}

While oil and biogenic film can cause dark signatures in synthetic aperture radar (SAR) images, exact quantification of the radar scattering damping mechanisms and the discrimination of slick areas from look-alikes are still challenging. The damping of short wind waves by surface films (biogenic and anthropogenic) has been subject to numerous experimental and theoretical studies (e.g., [1]-[12]). The resulting reduction of the normalized radar cross section (NRCS), $\sigma_{0}$, is usually associated to damping of resonant Bragg waves, providing polarized radar returns. However, Bragg scattering does not entirely control the returned signal power. As already well established (e.g., [13], [14]), non-polarized radar returns from breaking waves also play a significant role.

The earliest studies investigating the effects of surface films on short wind-waves and radar backscatter focused on a resonance-like damping behavior by monomolecular surface films resulting in a damping maximum predicted by the Marangoni damping theory (e.g., [6] and references therein). However, no damping maximum could be revealed in real slicks observed with SAR, ultimately indicating that wind forcing and wave breaking are key to

M. W. Hansen and J. A. Johannessen are with the Nansen Environmental and Remote Sensing Center, Bergen, Norway.

V. Kudryavtsev is with the Russian State Hydrometeorological University, Saint Petersburg, and the Marine Hydrophysical Institute, Sebastopol, Russia, and the Nansen Environmental and Remote Sensing Center, Bergen, Norway.

B. Chapron is with Ifremer-CERSAT, Brest, France.

C. Brekke is with the Department of Physics and Technology, University of Troms $\emptyset$ - The Arctic University of Norway, Troms $\varnothing$, Norway. 
control the spectral contrasts of waves in the slicks. [2]-[4], [7] suggested a model of the damping of the short wind wave spectrum, $E$, based on solution of the energy balance equation combining wind forcing, viscous dissipation (accounting for the Marangoni damping via the viscosity coefficient, $\nu$ ), and non-linear dissipation, $\varepsilon$, i.e.,

$$
\beta_{\nu} \omega E-\varepsilon+S_{w b}=0
$$

where $\beta_{\nu}=\beta-4 \nu k^{2} / \omega$ is an effective growth rate coefficient (i.e., the difference between the wind-wave growth rate coefficient, $\beta$, and the rate of viscous dissipation), and $\omega$ and $k$ are frequency and wavenumber linked by the dispersion relation. For the sake of generality, Eq. (1) also includes a term, $S_{w b}$, describing an additional energy source for short waves as suggested by [15].

[2], [3] assumed that non-linear dissipation is proportional to the squared spectrum, $E^{2}$, and found that the wave spectrum contrast between the slick-free and -covered areas (subscripts 0 and $S$, respectively) is $K \equiv E_{0} / E_{S}=$ $\beta_{\nu 0} / \beta_{\nu S}$. Upon testing this relation by optical measurements of short-wave spectral contrasts, an overall agreement was obtained between model and measurements in the decimeter wavelength range. However, a remarkable model overestimation was reported in the centimeter to millimeter wavelength range.

Employing Eq. (1), [4], [7] then suggested another expression, $K=\left(\beta_{\nu S} / \beta_{\nu 0}\right)\left(\varepsilon_{0} / \varepsilon_{S}\right)$, where $\varepsilon \propto E^{n}$ and $E \propto\left(u_{*} / c\right) k^{-4}$ (where $u_{*}$ is the friction velocity, and $c$ is the phase velocity). [7] assumed that the wave breaking dissipation exponent $n$ and the friction velocity, $u_{*}$, are both different in slick-covered areas compared to slickfree areas, i.e., $n_{0}$ versus $n_{S}$, and $u_{* 0}$ versus $u_{* S}$. Under this assumption, [7] showed that such a wave damping model, incorporated with the Bragg scattering model, is capable of reproducing observed multi-frequency and multipolarization NRCS contrasts of various surface slicks at high wind speeds $\left(12 \mathrm{~m} \mathrm{~s}^{-1}\right)$. However, the consistency was attained by adjusting the model parameters, $n_{0}-n_{S}$ and $u_{* S} / u * 0$, to match the observed contrast drop to each of the datasets. Although this model was successfully fitted to the data, no physical grounds justifying its applicability to other datasets was provided.

Except for L-band, the Bragg resonant scattering model represents an idealization that is too strong to fully explain radar backscattering in real conditions. As evident from polarization ratio (PR), $\sigma_{0}^{h h} / \sigma_{0}^{v v}$, measurements (e.g., [13], [14]), non-polarized scattering from breaking waves significantly contribute to the sea surface NRCS of $\mathrm{C}-$, X- and Ku-bands. Moreover, in addition to wind forcing, mechanical disturbances of the sea surface by breaking crests may serve as energy sources of short Bragg waves. As such, wave breaking thus plays a crucial role for the manifestation of various ocean parameters in SAR (including surface slicks), as justified and demonstrated in [15].

Dual co-polarized SAR measurements (VV and $\mathrm{HH}$ polarizations) have provided the opportunity to separate resonant Bragg scattering (via the polarization difference (PD), $\Delta \sigma_{0}=\sigma_{0}^{v v}-\sigma_{0}^{h h}$ ) and non-polarized (NP) scattering from breaking waves, and to investigate separately the responses to sea surface non-uniformities, i.e., surface currents, wind field features and sea surface slicks [16], [17]. Using Radarsat-2 C-band SAR data, [16] did not reveal any effect of surface film on the NP scattering from breaking waves. In comparison, [8] investigated the expressions of slick features in both TerraSAR (X-band) and Radarsat-2 (C-band) SAR images. As expected, the NP contribution to the total NRCS was higher in X-band than in C-band. In contrast to [16], however, [8] reported significant suppression of the NP radar backscatter in the presence of surface films. Recently, [18] suggested a 
TABLE I

MAIN CHARACTERISTICS OF THE RADARSAT-2 QUAD-POLARIZATION SAR DATA USED IN THE STUDY.

\begin{tabular}{|c|c|c|c|c|c|c|}
\hline ID & $\begin{array}{c}\text { Date } \\
\text { [Y-M-D H:M] }\end{array}$ & $\theta$ & $\begin{array}{c}U \\
{\left[\mathrm{~m} \mathrm{~s}^{-1}\right]}\end{array}$ & $\phi-\phi_{R}$ & $\mathrm{PR} / p_{B}$ & $\frac{\mathrm{CP}}{\mathrm{PD}} /\left(\frac{\mathrm{CP}}{\mathrm{PD}}\right)_{B}$ \\
\hline 1 & 2008-12-05 17:15 & $30^{\circ}$ & 5.1 & $320^{\circ}$ & $0.73 / 0.47$ & $0.022 / 0.009$ \\
\hline 2 & $2010-05-15 \quad 11: 56$ & $30^{\circ}$ & 6.4 & $199^{\circ}$ & $0.71 / 0.48$ & $0.020 / 0.010$ \\
\hline 3 & 2011-06-08 05:59 & $47^{\circ}$ & 6.3 & $225^{\circ}$ & $0.23 / 0.16$ & $0.028 / 0.010$ \\
\hline 4 & 2011-06-08 17:28 & $35^{\circ}$ & 4.5 & $15^{\circ}$ & $0.59 / 0.35$ & $0.018 / 0.009$ \\
\hline 5 & 2012-06-15 06:20 & $31^{\circ}$ & 4.3 & $89^{\circ}$ & $0.66 / 0.44$ & $0.021 / 0.009$ \\
\hline 6 & 2012-06-15 $\quad 17: 49$ & $49^{\circ}$ & 8.0 & $325^{\circ}$ & $0.31 / 0.14$ & $0.031 / 0.011$ \\
\hline 7 & 2013-06-11 $\quad 17: 20$ & $29^{\circ}$ & 6.8 & $126^{\circ}$ & $0.70 / 0.51$ & $0.021 / 0.010$ \\
\hline
\end{tabular}

new algorithm for discrimination of oil spills and look-alikes in dual co-polarized SAR measurements, using the observed effect of surface films on suppression of both Bragg waves and wave breaking.

In this paper, we follow the physically-based approach suggested in [16] and [15] to investigate and quantify the damping sensitivity of both centimeter-scale waves and longer breaking waves in the presence of known slicks, and to assess the relative contribution of Bragg-resonant and NP scattering in the formation of co- and cross polarized (HH, VV, and CP) NRCS contrasts between the clean and slick covered sea surface. In section II, the background properties and approach are introduced. The results are presented in section III and discussed in section IV. The conclusion is provided in section $\mathrm{V}$.

\section{BACKGROUND PROPERTIES AND APPROACH}

The Radarsat-2 quad-polarization SAR acquisitions used in the study are listed in Table I together with information on radar parameters and environmental conditions. In addition to data from the Norwegian Clean Seas Association for Operating Companies (NOFO) oil-on-water experiments at the Frigg field in 2011, 2012, and 2013 (with known film types, e.g., [8], [19]; ID's 3-7), one acquisition from the North Sea in 2008 (ID 1), with three slicks of unknown origin, and one acquisition of the oil spill from the Deepwater Horizon (DWH) accident in the Gulf of Mexico in 2010 (ID 2) are also included. By decomposing these Radarsat-2 SAR observations into polarization ratio (PR), polarization difference (PD), non-polarized (NP), and cross-polarized (CP) components, new quantitative insight regarding the damping of short waves and breaking waves in the presence of slicks is achieved. The estimation and characteristics of these components are further addressed in the following.

Since the C-band PR deviates remarkably from Bragg scattering predictions (e.g., [13], [14]), an additional NP contribution, $\sigma_{w b}$, associated with radar backscatter from breaking waves is introduced to yield

$$
\sigma_{0}^{p p}=\sigma_{0 B}^{p p}+\sigma_{w b}
$$

where $\sigma_{0 B}^{p p}$ is the two-scale Bragg scattering at given polarization ( $p$ ) expressed as

$$
\sigma_{0 B}^{p p}=\pi \sin ^{-4} \theta\left|G_{p p}(\theta)\right|^{2} B\left(\underline{\mathbf{k}}_{B}\right)\left(1+g_{p p} s_{i}^{2}\right),
$$


where $\left|G_{p p}(\theta)\right|$ is the scattering coefficient (e.g., Eqs. (3) and (4) in $\underline{[13]}$ ), $B\left(\underline{\mathbf{k}}_{B}\right)$ is the saturation spectrum at the Bragg wavenumber, $\left|\underline{\mathbf{k}}_{B}\right|=2 k_{R} \sin \theta, k_{R}$ is the radar wavenumber, and

$$
g_{p p}=\frac{\sin ^{4} \theta}{2\left|G_{p p}(\theta)\right|^{2}} \frac{\partial^{2}}{\partial \theta^{2}}\left(\frac{\left|G_{p p}(\theta)\right|^{2}}{\sin ^{4} \theta}\right)
$$

is a geometric coefficient accounting for the impact of tilting waves in the spectral range $k<k_{d}=k_{B} / 4$. $s_{i}^{2}$ is the mean square slope (MSS) of tilting waves in the direction of the incidence plane. $s_{i}^{2}$ can be evaluated as half of the total MSS, i.e., $s_{i}^{2}=s^{2} / 2$ prescribed empirically by [20] as $s^{2}=4.6 \times 10^{-3} \ln \left(k_{d} u_{10}^{2} / g\right)$, where $u_{10}$ is the wind speed at 10 meter height and $g$ is the gravitational acceleration. Note that the cross-polarized effect on $g_{p p}$ is ignored (see [13], Fig. 5), and that $B$ is a slowly varying function of $k$, as compared to $\left|G_{p p}(\theta)\right|^{2} / \sin ^{4} \theta$, and thus does not impact $g_{p p}$.

Following [13],

$$
\sigma_{w b}=\int_{k<c_{R} k_{R}} \sigma_{0 w b} \mathrm{~d} q,
$$

where $c_{R}$ is a constant (about 0.1 ), and $\sigma_{0 w b}$ is the NRCS of an "individual" breaking zone $\mathrm{d} q$, defined as

$$
\sigma_{0 w b} \propto\left(\sec ^{4} \theta / s_{w b}^{2}\right) e^{-\tan ^{2} \theta / s_{w b}^{2}},
$$

where $s_{w b}^{2}$ is the MSS of the breaking zone and $\theta=\theta_{0}+\theta^{\prime} . \theta_{0}$ is the radar incidence angle and $\theta^{\prime}=\bar{\theta}_{w b} \cos \left(\phi-\phi_{R}\right)$ is the local tilt, where $\bar{\theta}_{w b}$ is the mean tilt of the breaking zone, and $\left(\phi-\phi_{R}\right)$ is the wind direction relative to the radar look direction. Following [21], a breaking zone, $\mathrm{d} q$, can be expressed as $\mathrm{d} q \propto \beta B \mathrm{~d} \phi \mathrm{d} \ln k$, where $\beta$ is the wind wave growth rate. [13] inferred $s_{w b}^{2}=0.19$ as a unique constant for breaking waves at all scales. This assumption is revised in section IV to interpret the reported observations.

The polarization difference (PD), i.e.,

$$
\Delta \sigma_{0}=\sigma_{0}^{v v}-\sigma_{0}^{h h}=\sigma_{0 B}^{v v}-\sigma_{0 B}^{h h},
$$

best isolates the polarized contributions. Since PD is proportional to $B\left(k_{B}\right)$, which has a short relaxation time, the PD-signal follows the wind field variability and efficiently traces the surface film. Thus, the attenuation of PD in slicks directly relates to a damping of the Bragg wave spectrum.

The NP contribution to the NRCS [16] becomes

$$
\sigma_{w b}=\sigma_{0}^{v v}-\Delta \sigma_{0} /\left(1-p_{B}\right)
$$

where $p_{B}=\sigma_{0 B}^{h h} / \sigma_{0 B}^{v v}$ is the polarization ratio for the two-scale Bragg scattering, depending on the incidence angle, $\theta$, and (weakly) on the wind speed via $s_{i}^{2}$ in Eq. (3). The influence of uncertainties in the definition of $p_{B}$ is discussed in [17].

Comparisons of the measured PR with the two-scale Bragg polarization ratio, $p_{B}$, calculated using Eq. (3) are listed in Table I and plotted in Fig. 1(a). As expected, the measured PR remarkably deviates from the Bragg predictions, $p_{B}$, due to significant contribution of non-polarized returns to the sea surface NRCS, Eq. (1). The relative contribution of NP returns (Eq. 8) to the total NRCS, $\sigma_{w b} / \sigma_{0}^{p p}$ for the data shown in Table I, is plotted 

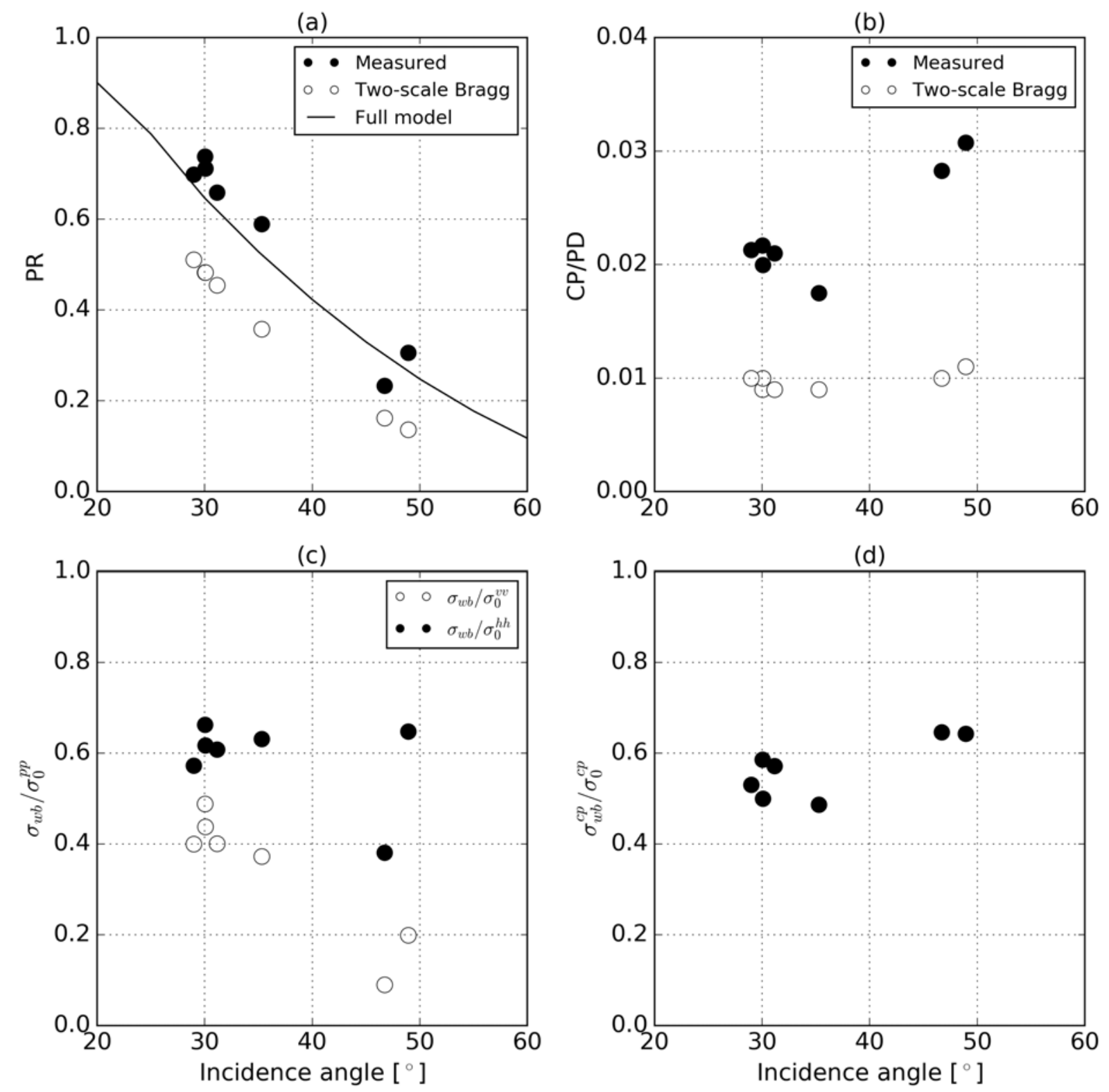

Fig. 1. Background (clean sea surface) properties of the quad-polarization SAR signals; (a) measured and modeled PRs, (b) measured and modeled CP-to-PD ratios, (c) relative contribution of wave breaking to the $\mathrm{HH}$ and VV NRCS, and (d) relative contribution of wave breaking to the CP NRCS.

in Fig. 1(c). As expected, the relative contribution of the NP returns is larger in $\mathrm{HH}(60 \%)$ than in VV (20-40\%, depending on incidence angle).

The last column of Table I show comparisons of measured CP-to-PD ratios with two-scale Bragg model predictions. To the first order of the MSS of tilting waves, two-scale Bragg CP reads [22]:

$$
\sigma_{0 B}^{c p}=\pi \sin ^{-6} \theta\left|G_{v v}(\theta)-G_{h h}(\theta)\right|^{2} s_{n}^{2} B\left(\underline{\mathbf{k}}_{B}\right),
$$


where $s_{n}^{2}$ is the component of the MSS of tilting waves out of the incidence plane $\left(s_{n}^{2}=s^{2} / 2\right.$, as for $\left.s_{i}^{2}\right)$. Comparing Eq. (9) with Eq. (3) one may conclude that Bragg CP-to-PD ratio does not depend on the Bragg wave spectrum, but only the MSS component out of the incidence plane. However, consistent with [17], the observed CP-to-PD ratio exceeds the two-scale Bragg model predictions by a factor of 2 to 3 (see Fig. 1). Even under moderate wind conditions, this indicates a significant contribution of wave breaking to the CP NRCS.

Similar to co-polarized measurements, this suggests a practical decomposition of the CP NRCS between a regular surface contribution described by the two-scale Bragg model and radar backscatter from rougher surface patches associated with breaking waves, i.e., following [17],

$$
\sigma_{0}^{c p}=\sigma_{0 B}^{c p}+\sigma_{w b}^{c p}
$$

The quad-polarization SAR data allows assessing the wave breaking contribution $\sigma_{w b}^{c p}$. From Eq. (10) we have:

$$
\sigma_{w b}^{c p}=\sigma_{0}^{c p}-r_{B} \Delta \sigma_{0}
$$

where $r_{B}=\sigma_{0 B}^{c p} / \Delta \sigma_{0}$ is the CP-to-PD ratio in the two-scale Bragg model. Fig. 1 shows the ratio between the wave breaking contribution to $\mathrm{CP}$ and the total CP NRCS, $\sigma_{w b}^{c p} / \sigma_{0}^{c p}$ calculated using Eq. (11) with the numbers obtained in Table I. The contribution of wave breaking to the total CP NRCS varies from $50 \%$ to $65 \%$, i.e., it is comparable to the Bragg scattering contribution.

To simulate $\sigma_{w b}^{c p}$ we follow the approach used for $\sigma_{w b}$. As suggested, the wave breaking contribution, $\sigma_{w b}^{c p}$, can follow a relation similar to Eq. (5), using $\sigma_{0 w b}^{c p}$ as the CP NRCS of an individual breaking zone. As quasi-specular reflections do not directly lead to depolarized radar backscatter, a heuristic model for $\sigma_{0 w b}^{c p}$ can still follow a resonant Bragg curvature approximation [22]. In this case the CP NRCS for individual breaking areas reads [17]

$$
\sigma_{0 w b}^{c p}=\pi \sin ^{-4} \theta\left|G_{v v}(\theta)-G_{h h}(\theta)\right|^{2} B_{b}\left(k_{B}\right) \theta_{n}^{\prime 2} / \sin ^{2} \theta
$$

where $G_{v v}(\theta)$ and $G_{h h}(\theta)$ are the standard Bragg scattering coefficients, $B_{b}$ is the saturation spectrum of the breaking crest roughness (defined below in section IV), and $\theta_{n}^{\prime}=\bar{\theta}_{w b} \sin \left(\phi-\phi_{R}\right)$ is the mean tilt out of the incidence plane of the breaking zone. The integral over all breaking zones, $d q$, provides the total contribution of wave breaking to the CP NRCS, i.e.,

$$
\sigma_{w b}^{c p}=\int_{k<c_{R} k_{R}} \sigma_{0 w b}^{c p} \mathrm{~d} q .
$$

In the present study we use the short wind wave model spectrum suggested in [23], which represents a minor revision of the spectral model used in the radar imaging model presented in [15]. The short-wave spectrum results from a solution of the energy balance equation (similar to Eq. (1)), which takes into account generation of short waves by wave breaking, i.e., the term $S_{w b}$ (see (Eqs. [A1] and [A4] in [23]) for more details), expressed as

$$
S_{w b}(k) \propto \omega^{2} k^{-5} \int_{k<k_{m}} \omega \mathrm{d} q,
$$

where $k_{m}=\min \left(k / 10, k_{w b}\right)$ defines the wavenumber interval of the long breaking waves from which energy is transferred to the short-waves, and $k_{w b}$ is on the order of $10 \mathrm{rad} \mathrm{m}^{-1}$. 

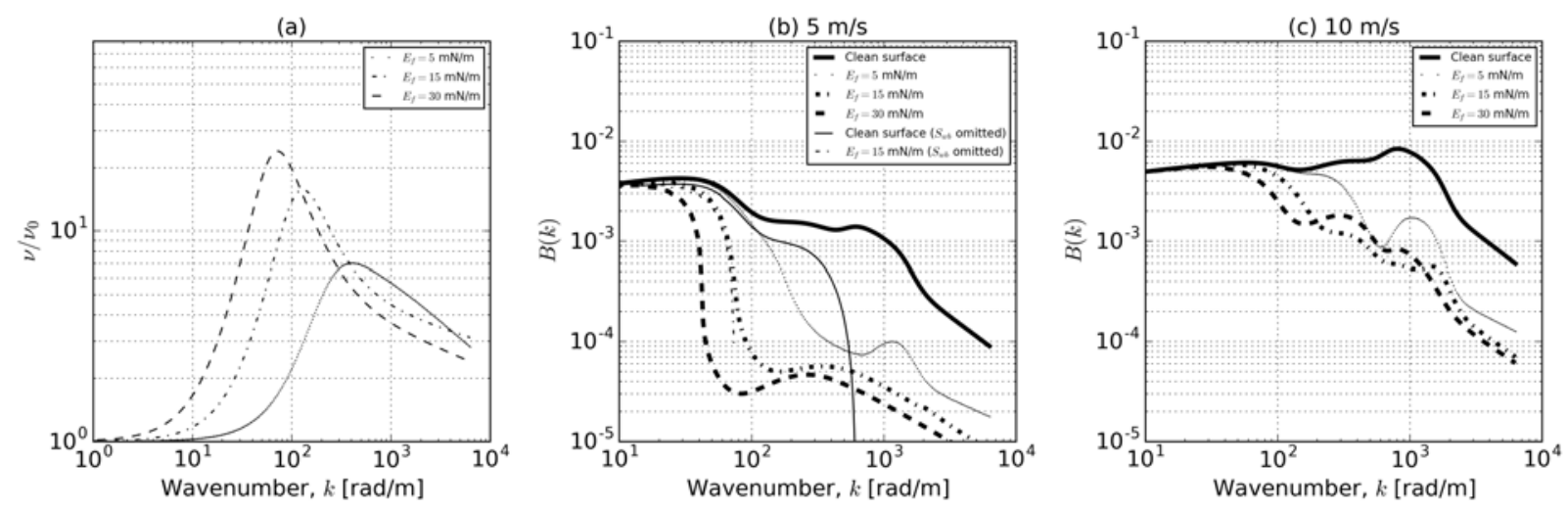

Fig. 2. (a) Simulated influence of various surface film elasticities on the effective viscosity coefficient scaled by the viscosity of clean water, and $(\mathrm{b}, \mathrm{c})$ omni-directional saturation spectra, $B(k)$, of short wind-waves at wind speeds of (b) $5 \mathrm{~m} \mathrm{~s}^{-1}$ and (c) $10 \mathrm{~m} \mathrm{~s}^{-1}$. The thin solid and dash-dotted lines in (b) are spectra when $S_{w b}$, Eq. (14), is omitted.

As in [2], [4], the damping of short wind-waves in the slicks is estimated by replacing the water viscosity, $\nu_{0}$, in the energy balance equation (see [23]) by an effective viscosity coefficient, $\nu$. The ratio $\nu / \nu_{0}$ depends on the film elasticity, $E_{f}$, generally accepted to vary from 15 to $45 \mathrm{mN} \mathrm{m}^{-1}$ for different biogenic films at the ocean surface (e.g., [2], [4], [15]). For mineral oil, S. Ermakov (personal communication) suggested to use $E_{f}=4 \mathrm{mN} \mathrm{m}^{-1}$, whereas [24] found that $E_{f}=15 \mathrm{mN} \mathrm{m}^{-1}$ provides the best agreement between simulated and observed mean square slope in crude oil slicks.

Fig. 2(a) shows the simulated influence of various surface film elasticities on the effective viscosity coefficient, $\nu / \nu_{0}$. Two types of model spectral contrasts are shown in Fig. 2(b). Model results from the truncated energy balance Eq. (1), where the term $S_{w b}$ is omitted (this model is similar to the one considered by [2]), are shown in thin lines for a clean and a slick covered sea surface. In this case, the spectral contrasts exhibit a spectral cut-off at the wavenumbers where $\beta_{\nu}=0$. At larger wavenumbers, the wind energy input is less than the viscous dissipation, and thus the short waves disappear. If the extra energy source, $S_{w b}$, defined by Eq. (14) is taken into account in Eq. (1) (see also Eq.[A1] and [A4] in [23] for details), the energy flux from larger-scale breaking waves to short waves enhances the spectral level in slick-free conditions, and prevents the disappearance of short waves in the slicks (thick lines). In this case, the film also cause a spectral cut-off around $k=30 \mathrm{rad} \mathrm{m}^{-1}$, but the energy source $S_{w b}$ keeps the short-wave spectrum in the slicks at a "finite" level.

Fig. 2(c) and (d) show the omni-directional saturation spectra, $B(k)$, of short wind-waves at different film elasticities, $E$, and wind speeds for the full model. The spectral "cut-off" depends on $E_{f}$ and the wind speed (e.g., about 20 to $60 \mathrm{rad} \mathrm{m}^{-1}$ and 30 to $100 \mathrm{rad} \mathrm{m}^{-1}$ for 5 and $10 \mathrm{~m} \mathrm{~s}^{-1}$ wind, respectively). Unless $k_{B}$ gets into the interval between the cutoff wavenumbers for films of different types, the contrast between the slick and the clean surface in the Bragg NRCS, $\sigma_{0 B}^{p p}$, should not be sensitive to the type of film. For wind speeds of $5 \mathrm{~m} \mathrm{~s}^{-1}$ and $10 \mathrm{~m} \mathrm{~s}^{-1}$, respectively, $k_{B}$ comes within this interval at incidence angles of $\theta<15^{\circ}$ or $\theta<26^{\circ}$ for a C-band radar. However, at such incidence angles, the sea surface NRCS is mostly supported by specular reflections, and the 
contribution of Bragg scattering to the total NRCS is small or negligible. In the range of incidence angles $\theta>30^{\circ}$ considered in this study, the Bragg scattering (represented by PD, Eq. (6)) should not be sensitive to the film type.

Note that, according to the model simulations shown in Fig. 2, the wave spectrum in the wavenumber range providing NP radar backscatter $\left(k<k_{R} / 10 \approx 11 \mathrm{rad} \mathrm{m}^{-1}\right.$, see [13] $)$ is insensitive to damping by any of the film types. This means that wave breaking (and consequently NP) should not be damped in the slicks. However, this is in contradiction to the observed suppression of NP radar backscatter reported by [8]. Therefore, another mechanism, different from the "simple" suppression of the spectral level, should be responsible for the impact of surface film on wave breaking.

\section{Results}

Acquisitions no. 4 on 8 June 2011 and no. 5 on 15 June 2012 are shown in Figs. 3 and 4, respectively. Scene no. 4 contains three different surface slicks, including plant oil, water-in-oil emulsion, and crude oil. Scene no. 5 contains two slicks of plant oil and emulsion. The co- and cross-polarized (CP, hereinafter defined as $\left.\sigma_{0}^{c p}=\left(\sigma_{0}^{h v}+\sigma_{0}^{v h}\right) / 2\right)$ NRCS (in linear units) were smoothed by the Lee adaptive filter to reduce speckle noise, and the Noise Equivalent Sigma Zero (NESZ), provided with the SAR product (see, e.g., [25]), was subtracted. The slicks are visible as dark areas in the HH, VV, CP, PD and NP images. In the PR image, on the other hand, the slicks are visible as bright areas. Another area in the lower left part of scene no. 4 is also dark in the HH, VV, PD, and NP images, and in scene no. 5 there is a dark region in the upper left. Due to similarity with true slicks, these areas could erroneously be associated with oil spills. However, the $\mathrm{PR}\left(\sigma_{0}^{h h} / \sigma_{0}^{v v}\right)$ values in these areas are significantly lower than the PR values in the slicks (approaching 1 due to dominant NP radar backscatter). As such, the areas are probably not covered by slick but, consistent with findings reported by [16], are regions of reduced wind. As wave breaking is strongly wind dependent, the slicks and the low wind feature are also well expressed in the NP images. However, since waves of about $0.6 \mathrm{~m}$ and longer ( $\left.k<k_{R} / 10\right)$ should not be damped (see Fig. 2), the measurable attenuation of the NP-signal in the slicks is thus more surprising.

In Figs. 5 and 6 (upper panels), the filtered co- and cross-polarized NRCS across the three slicks in scene no. 4 and the two slicks in scene no. 5 are compared to the NESZ. All signals are here above the NESZ level, even in the slick areas, and this is also the case for the other acquisitions considered in the study. As such, the filtering and subsequent NESZ subtraction should be sufficient to yield consistent noise corrected data for further analysis. In the lower panels, the contrasts of the SAR quantities (HH, VV, PD, NP, PR) across the different slicks are shown. The contrast of a quantity $Y$ is equivalent to the signal modulation in the slick, and is defined as the ratio between its averaged ambient value, $\bar{Y}$, and its local value $Y(x): K=\bar{Y} / Y(x)$. The largest contrast, observed in $\mathrm{PD}$, is up to twice as large as the VV contrast, whereas the HH contrast is significantly smaller than the VV one. This is in line with the increased PR in the slick areas (see Figs. 3 and 4). Although much lower than the PD contrasts, the NP ones are surprisingly significant. Furthermore, whereas the PD and CP contrasts should be comparable within a two-scale Bragg framework, the observed CP contrast is remarkably smaller than that of the PD. Interestingly, the $\mathrm{CP}$ contrasts are more comparable to the NP ones. Note, however, that if the magnitude and shape of the CP 

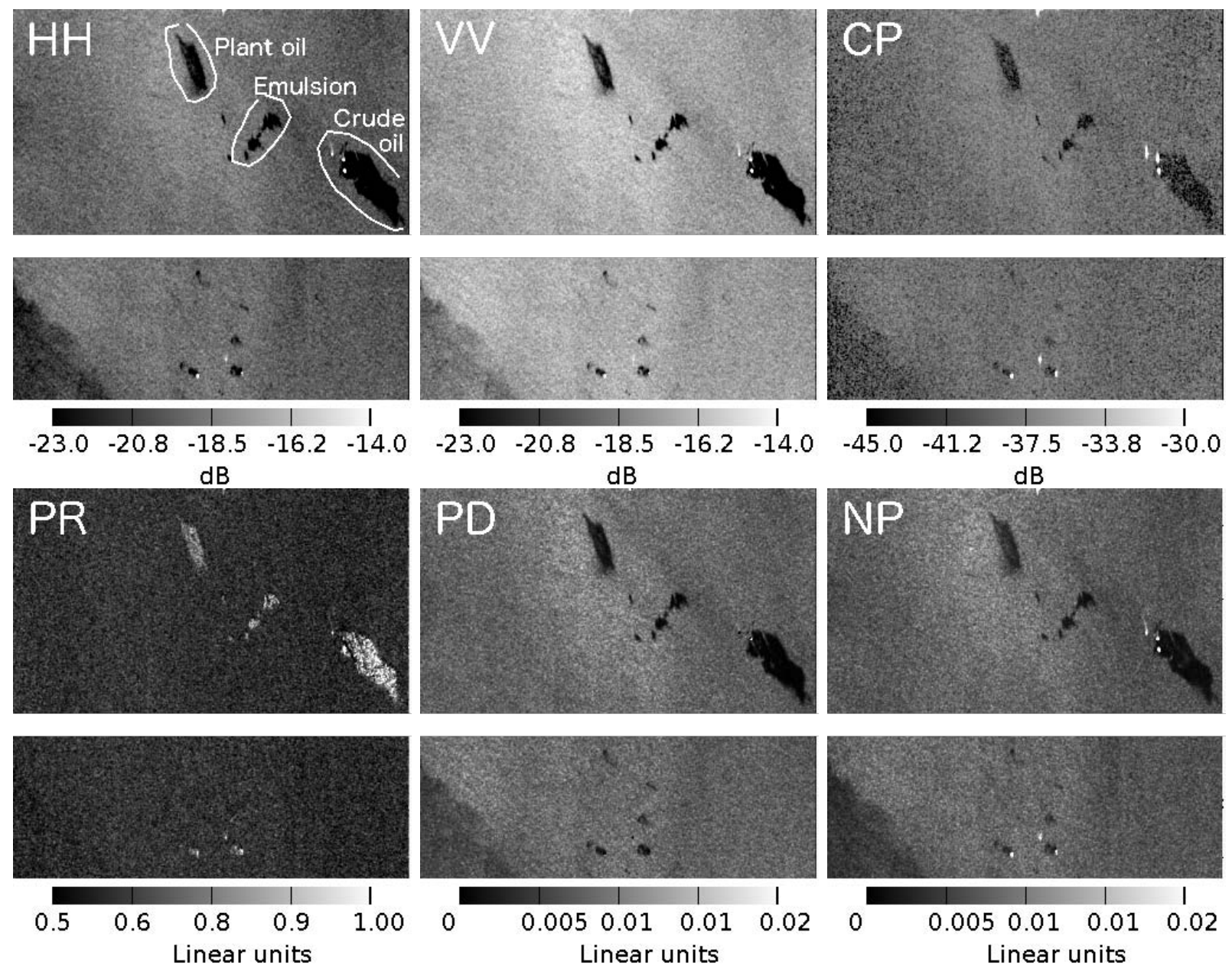

Fig. 3. Co-polarization (HH and VV), cross-polarization (CP), polarization ratio (PR), polarization difference (PD), and non-polarized (NP) images from the Radarsat-2 acquisition on 8 June 2011 at 17:28. The three slicks in the upper right corners are plant oil (left), emulsion (center), and crude oil (right). Note that the central part of each image has been removed due to its uniform backscatter.

contrast transects are very different from that of the other parameters, this could indicate noisy data. Such CP measurements, including the one for crude oil in Fig. 5, are therefore discarded in the following analysis.

The wind speed dependence of the VV, HH, and CP contrasts and the contrasts of their PD, NP, and CPwb derivatives are shown in Fig. 7 for all the scenes listed in Table I. As expected, the suppression of the VV NRCS in slicks is stronger than the suppression of the HH NRCS. This is confirmed by the increased PR in the slicks as compared to the ambient areas, see Figs. 3 and 4. The largest contrasts are observed in PD, while suppression of the NP radar backscatter associated with wave breaking are observed in all cases, although (about $50 \%$ ) less than the suppression of the Bragg waves associated with the PD signal. As a consequence of significant wave breaking contribution to CP (see Fig. 1), the magnitudes of the CP contrasts are remarkably lower than the magnitudes of the $\mathrm{PD}$ contrasts. The contrasts of the $\mathrm{CP}$ component caused by scattering from breaking waves (CPwb) have magnitudes which are similar to the NP contrasts. The VV, HH, PD, and NP contrasts clearly decrease with increasing wind speed, whereas the CP measurements are somewhat more scattered with a less clear trend. After careful inspection 

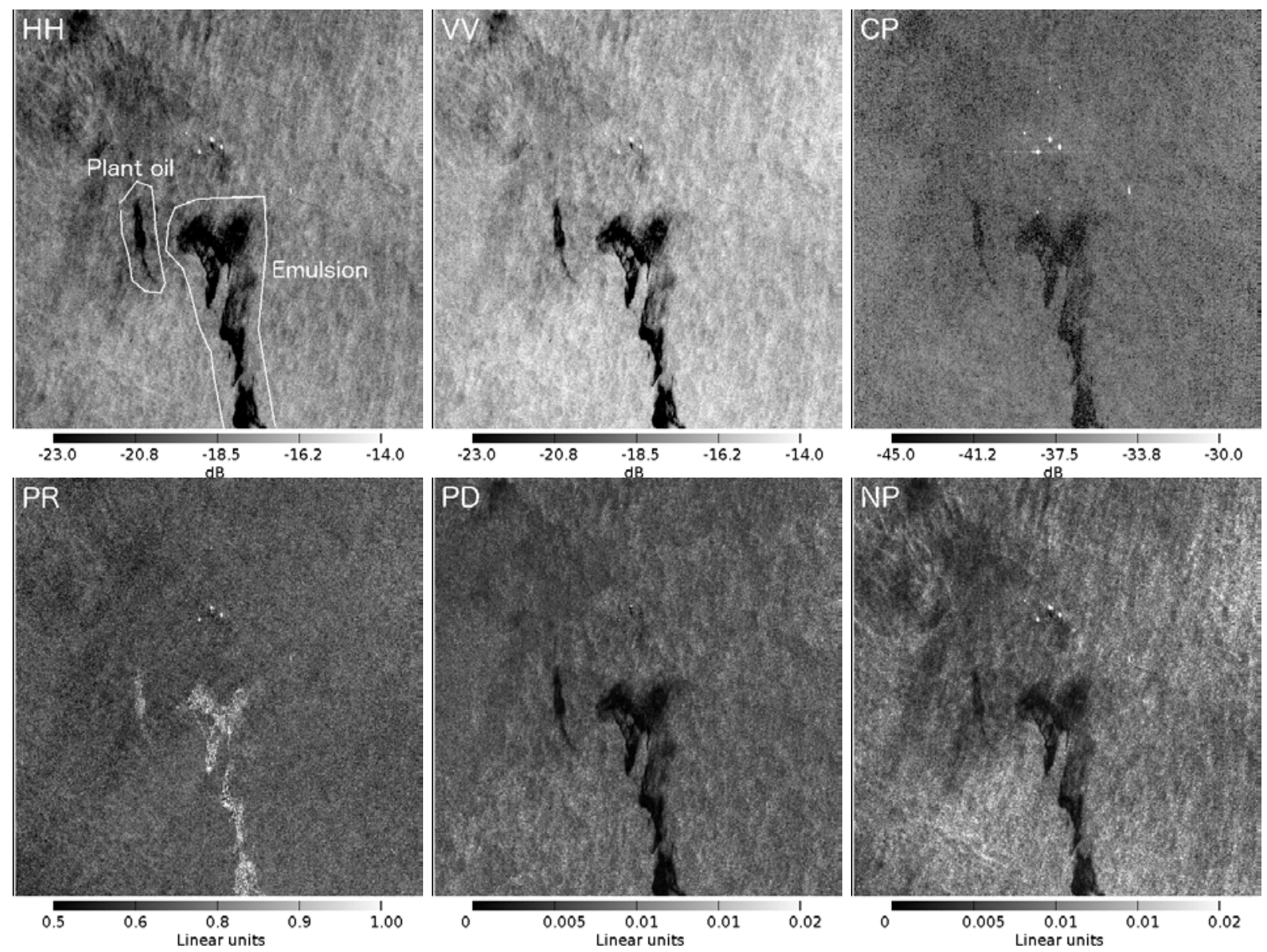

Fig. 4. Co-polarization ( $\mathrm{HH}$ and $\mathrm{VV})$, cross-polarization $(\mathrm{CP})$, polarization ratio (PR), polarization difference (PD), and non-polarized (NP) images from the Radarsat-2 acquisition on 15 June 2012 at 06:20. The slicks are plant oil and emulsion, as indicated in the HH image.

of Fig. 7, one may conclude that the contrasts are weakly dependent on the radar look-direction and that the crude oil and emulsion slicks appear to cause stronger wave damping than the plant oil slicks.

\section{DISCUSSION}

As already noticed, increased effective viscosity does not affect the spectral level in the wavenumber range of breaking waves $\left(k<0.1 / k_{R}\right)$ providing NP radar backscatter (see Fig. 2). Thus, we could anticipate that wave breaking is not changed in the slicks. However, this comes into conflict with the observed suppression of the NP and CPwb NRCS components in the slicks, see Fig. 7. In order to reconcile the model according to the observations, we hypothesize that the spectral level determines only the kinematic properties of the breaking waves (e.g., the number of breaking events per surface unit). As a consequence, the number of breaking waves per surface unit is not sensitive to the presence of film. On the other hand, we assume that surface film do affect the intensity of an individual breaking crest. The breaking crest intensity necessarily defines the MSS, $s_{s w}^{2}$, of the breaking crest roughness in Eq. (6) for NP, its spectrum, $B_{b}\left(k_{B}\right)$ in Eq. (12) for CPwb, and the area of the individual breaking 

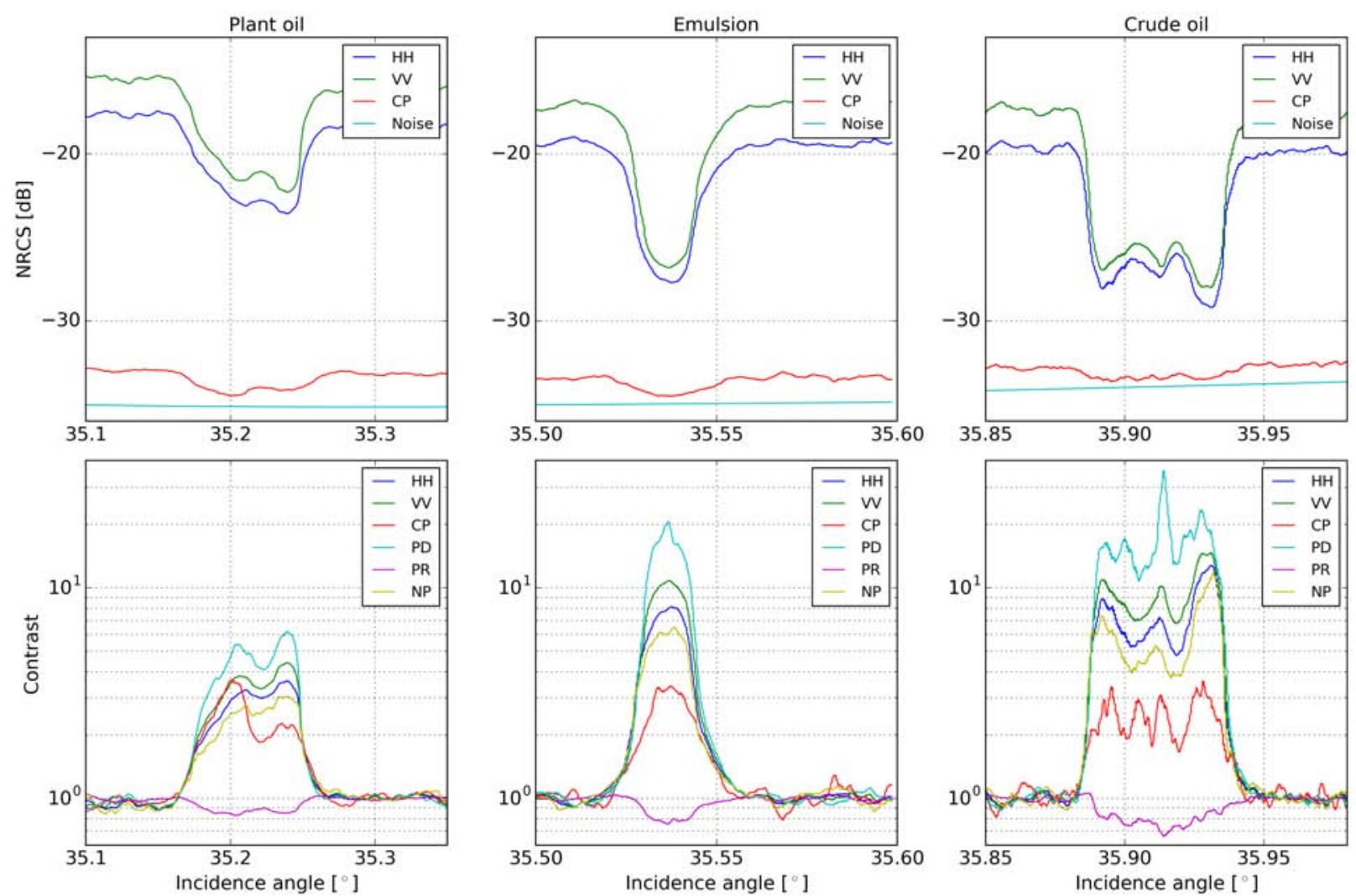

Fig. 5. VV, HH and CP NRCS in comparison to the NESZ across the three different slicks in acquisition 4 from 8 June 2011 at $17: 28$ (upper panels), and the contrasts between the slick and the ambient clean sea surface in the same transects for each SAR quantity (lower panels).

zone, $\mathrm{d} q$, that appears in each of the scattering properties, including the wave breaking source $S_{w b}($ Eq. (14)) which defines the Bragg waves spectrum.

From laboratory experiments [26], a 1D breaking crest roughness spectrum falls off at about $-5 / 2$ for wavenumbers larger than those of the individual breaking waves. Following [27], such a spectrum can be treated as a Kolmogorov-type spectrum generated by the energy flux, $P$, which cascades from the largest energetic breaking disturbances toward shorter ones, i.e.,

$$
P \propto \beta_{b} g \omega_{b} k_{b}^{-2} \propto \beta_{b} c_{b}^{3}
$$

where $\beta_{b}$ is the dimensionless growth rate of the crest instability, $\omega_{b}, k_{b}$ and $c_{b}$ are the frequency, the wavenumber, and the phase velocity of the breaking wave, respectively. From dimensional analysis, the omni-directional roughness spectrum generated by a breaking crest is

$$
\psi_{b}(k) \propto g^{-1} P^{2 / 3} k^{-2} \propto \beta_{b}^{2 / 3} k_{b}^{-1} k^{-2}
$$

For breaking waves of large scales compared to the radar wavelength, this leads to the relationship

$$
s_{w b}^{2}=b \beta_{b}^{2 / 3} k_{R} / k_{b}
$$



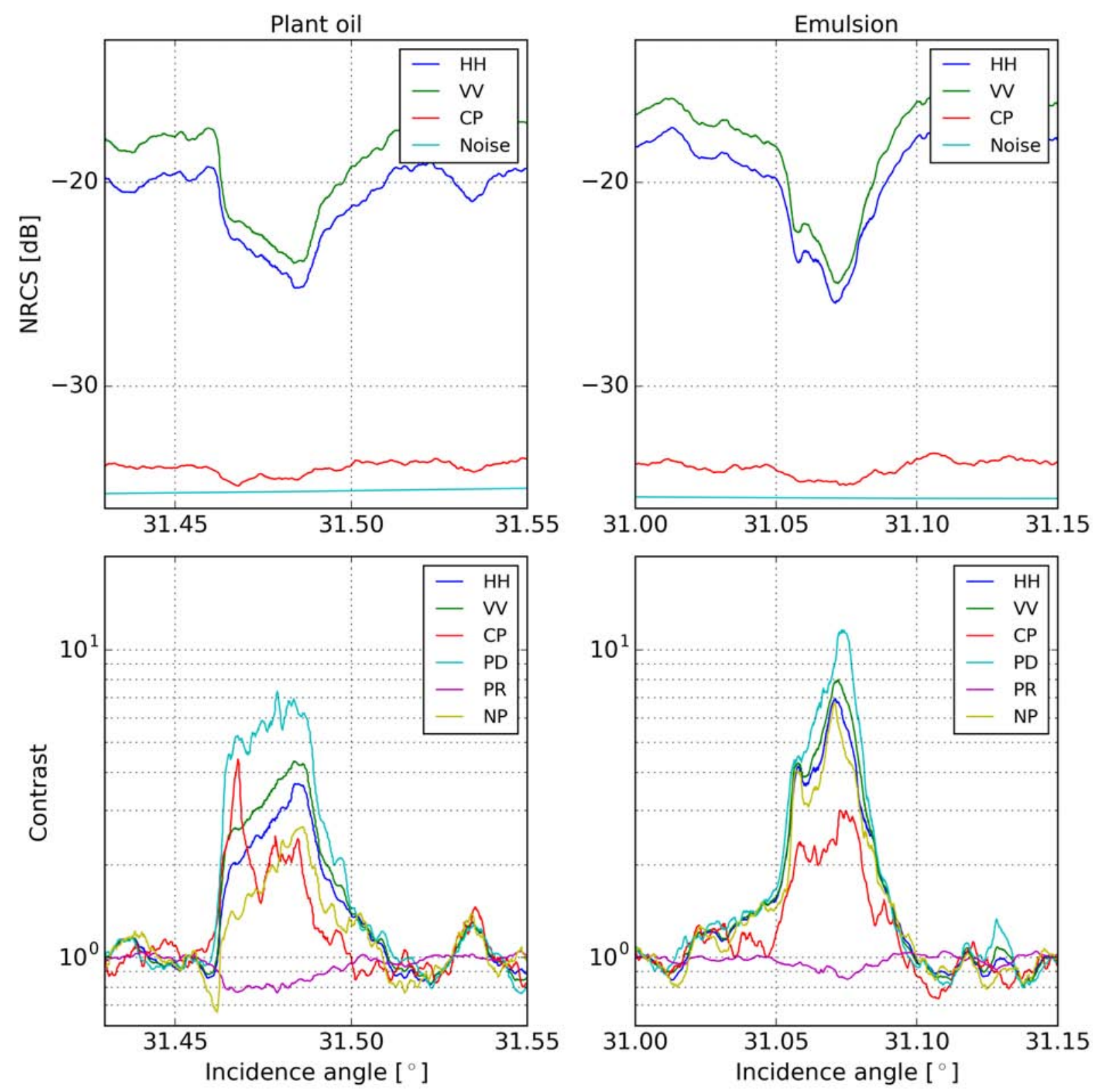

Fig. 6. VV, HH and CP NRCS in comparison to the NESZ across the two slicks in acquisition 5 from 15 June 2012 at 06:20 (upper panels), and the contrasts between the slick and the ambient clean sea surface in the same transects for each SAR quantity (lower panels).

for the breaker roughness MSS, which increases with the scale of the breaking waves. The constant $b$ in Eq. (17) is a tuning constant to adjust the NRCS model to the measured PRs listed in Table I, following the model parameterization of $\mathrm{d} q$ in Eq. (5) presented in [15] (see model PR in Fig. 1a). The other breaking roughness quantity, i.e., the saturation spectrum $B_{b}\left(k_{B}\right)$ needed for CPwb in Eq. (12), apparently follows from Eq. (16), and reads

$$
B_{b}\left(k_{B}\right) \propto \beta_{b}^{2 / 3} k_{B} / k_{b}
$$



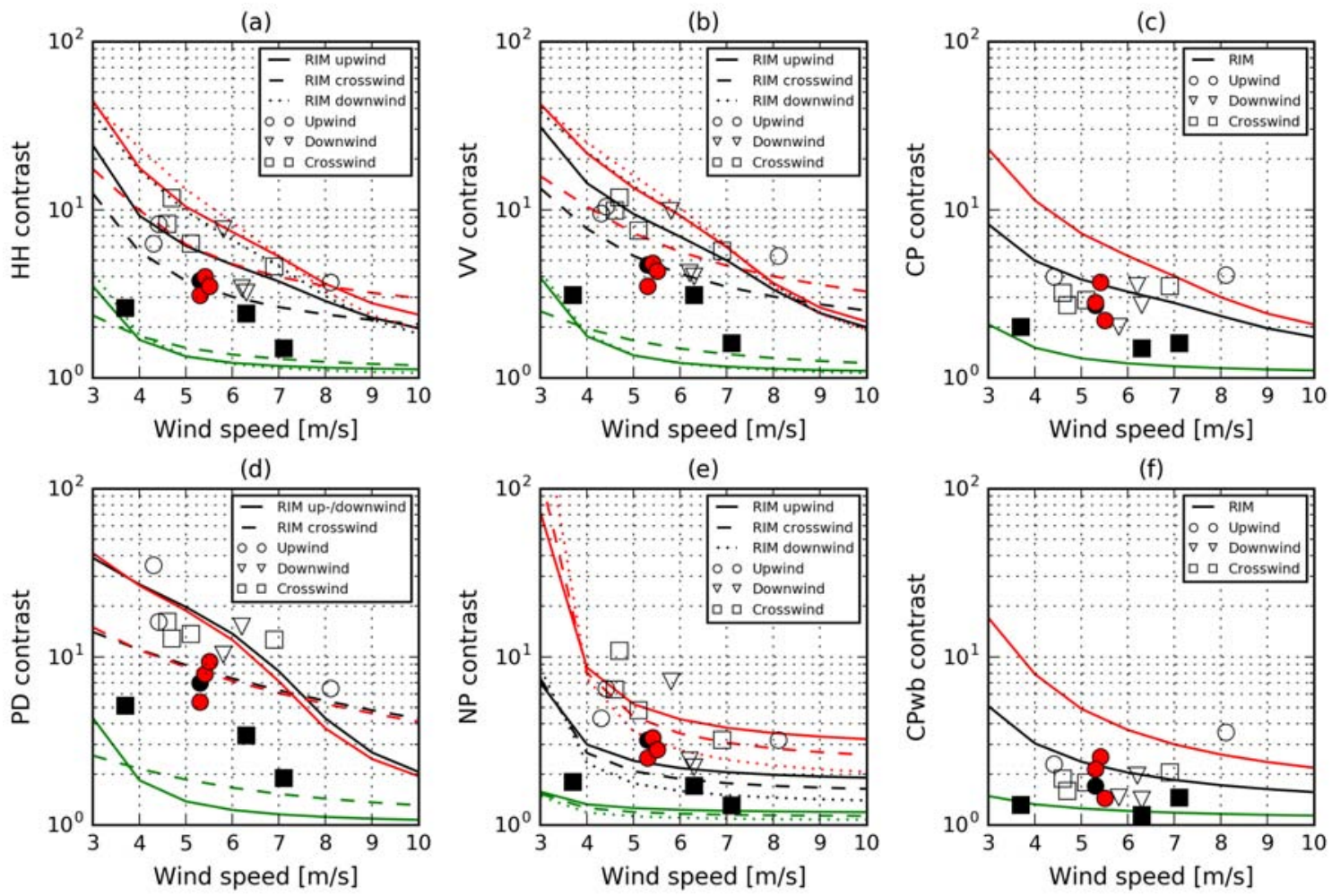

Fig. 7. Contrasts between the clean surface and the slicks versus wind speed for the observations and simulations of (a) HH, (b) VV, (c) CP, (d) PD, (e) NP, and (f) the wave breaking contribution to the CP NRCS. The open symbols are crude oil and emulsion, and the filled symbols are plant oil (black) and of unknown type (red). The curves represent the contrasts in up- (solid), down- (dotted) and crosswind (dashed) configurations, simulated with the Radar Imaging Model (RIM) at $35^{\circ}$ incidence angle with $E_{f}=5,15,30 \mathrm{mN} \mathrm{m}^{-1}$ (green, black and red). The simulation of the PD contrast is based on [13] and [23], whereas the simulations of the other quantities also take into account the model developments in section IV.

Furthermore, the area, $\mathrm{d} q$, of an individual breaking zone could also be reduced. The breaking area generated by the energy flux $P$ from large to smaller scales, can be deduced from the energy conservation equation

$$
g \omega h_{b}^{2} \mathrm{~d} q \propto P h_{b} \mathrm{~d} L
$$

where $\mathrm{d} L$ is the breaking crest length, and $h_{b}$ scales as the breaking zone thickness, defined from Eq. (16) as $h_{b} \propto \beta^{1 / 3} k_{b}^{-1}$. Thus, the area of the breaking zone is

$$
\mathrm{d} q \propto \beta^{2 / 3} k_{b}^{-1} \mathrm{~d} L
$$

Following [28], the dimensionless growth rate of an isolated and unstable gravity wave crest, scaled by the wave frequency, $\omega_{b}$, is $\beta_{b}=c_{i}\left(k_{b} R\right)^{-1 / 2}$, where $R$ is the radius of curvature at the undisturbed crest, and $c_{i}=0.123$. If the instability develops on a dimensionless time scale, $1 / \beta_{b}=1$, the initial crest curvature should be about $k_{b} R=0.015$. However, if it develops within half of a wave period, $1 / \beta_{b}=\pi, k_{b} R=0.15$.

An intense breaking wave should mechanically disrupt the surface film. As such, the film itself cannot strongly damp the spectral disturbances, Eq. (16). More likely, the surfactants suppress short harmonics that trigger the crest 
instability. For a highly curved clean crest, the spectrum of the short harmonics (that trigger crest instability) is proportional to $k^{-4}$. Thus, the radius of the crest curvature is, $1 / R=k_{m} / \pi$, where $k_{m}$ is the wavenumber of the shortest harmonic triggering the crest instability. With $m=k_{m} / k_{b}$, we have $\beta_{b}=c_{i}(m / \pi)^{1 / 2}$. Surface film can then effectively damp the energy of the shortest harmonics, $k<k_{m}$, with rate $\propto \nu k^{2}$, to restrain the development of the crest instability. This expected reduction of the crest instability can be taken into account as

$$
\beta_{b} \propto m^{1 / 2}\left(\int_{k_{b}}^{m k_{b}} \nu_{0} \mathrm{~d} \ln k\right) /\left(\int_{k_{b}}^{m k_{b}} \nu \mathrm{d} \ln k\right),
$$

where the integrals represent the rate of the harmonics energy dissipation due to viscosity for the clean and film covered surface. In this paper we set $m=20$ corresponding to $k_{b} R=0.15$. Crest instability of a large breaking wave can then be formed by harmonics well outside the spectral range where the difference between $\nu$ and $\nu_{0}$ comes into effect. In contrast, small breaking waves will be smoothed out and damped by the surfactants, to fully restrain the breaking intensity. For the clean surface $\nu / \nu_{0}=1$, and thus $\beta_{b 0} \propto m^{1 / 2}$.

Thus, the impact of the surfactants on the breaking crest MSS, Eq. (17), its spectrum around the Bragg wavenumber, Eq. (18), and the fraction of the surface covered by an individual breaking zone, Eq. (20), can be described using the modified crest instability $\beta_{b}$, defined by Eq. (21). As a result of the breaking wave parameters being affected by surface film, the radar backscattering components are also suppressed, i.e., $\sigma_{w b}\left(\right.$ Eqs. (5) and (6)), $\sigma_{w b}^{c p}$ (Eqs. (12) and (13)), and $\sigma_{0 B}^{p p}$ and $\sigma_{0 B}^{c p}$ (due to the impact of the film on the regular wave spectrum via the wave breaking source, $S_{w b}$, Eq. (14)).

To model the contrasts between the clean surface and the slicks, a damping factor describing the reduction of the breaking crest instability growth rate, Eq. (21), by the film is introduced, i.e.,

$$
D\left(k_{b}\right)=\beta_{b} / \beta_{b 0}
$$

where the subscript 0 stands for clean surface conditions. Then, the NP radar backscatter contrast between the clean surface and the slick reads

$$
K_{w b} \equiv \frac{\sigma_{w b 0}}{\sigma_{w b}}=\frac{\int_{k<c_{R} k_{R}} k^{-1} e^{-\tan ^{2} \theta / s_{w b 0}^{2} \mathrm{~d} q_{0}}}{\int_{k<c_{R} k_{R}} k^{-1} e^{-D^{-2 / 3} \tan ^{2} \theta / s_{w b 0}^{2} \mathrm{~d} q_{0}}} .
$$

Similarly, the wave breaking component of the CP NRCS contrast between the clean surface and the slick reads

$$
K_{w b}^{c p} \equiv \frac{\sigma_{w b 0}^{c p}}{\sigma_{w b}^{c p}}=\frac{\int_{k<c_{R} k_{R}} k^{-2} \mathrm{~d} q_{0}}{\int_{k<c_{R} k_{R}} D^{4 / 3} k^{-2} \mathrm{~d} q_{0}} .
$$

Suppression of wave breaking via the energy source $S_{w b}$ impacts (together with enhanced viscous dissipation, term $\beta_{\nu}$ in Eq. (1)) the energy balance of short waves, and thus the spectrum of Bragg waves. In the slick area, generation of short waves by larger-scale breaking is $S_{w b}=K_{w b}^{s w} S_{w b 0}$, where

$$
K_{w b}^{s w} \equiv \frac{S_{w b 0}}{S_{w b}}=\frac{\int_{k<k_{m}} \omega \mathrm{d} q_{0}}{\int_{k<k_{m}} D^{2 / 3} \omega \mathrm{d} q_{0}} .
$$


is the short-wave contrast. Solution of Eq. (A1) from [23] with a modified wave breaking source (Eq. (25)) and with enhanced viscous dissipation then describes the response of Bragg waves to the presence of film.

Fig. 7(d) represent the measured PD contrasts (equivalent to the Bragg wave spectrum contrasts) and their simulations using the spectrum model described in [23] at Bragg wavenumber corresponding to $\theta=35^{\circ}$. The model can reproduce the observed PD contrasts for crude oil and emulsion for $E_{f}$ in the range 15 to $30 \mathrm{mN} \mathrm{m}^{-1}$, whereas the observed PD contrasts in plant oil slicks could be reproduced with lower $E_{f}$ in the range 5 to 15 $\mathrm{mN} \mathrm{m}^{-1}$.

The modeled contrasts, $K_{w b}$ and $K_{w b}^{c p}$, between the clean surface and slicks for the NP, Eq. (23), and the wave breaking contribution to CP, Eq. (24), are shown in Figs. 7 (e) and (f), respectively. In line with [24], who estimated $E_{f}=15 \mathrm{mN} \mathrm{m}^{-1}$ for mineral oil slicks, the simulated contrasts in up-, cross-, and downwind configurations for NP and CPwb radar backscatter, as well as their wind dependency, agree well with the observations.

Note that the observed values of $K_{w b}^{c p}$, shown in Fig. 7(f), are estimated from the measured contrasts of CP and PD signals using

$$
K_{w b}^{c p}=\frac{K^{c p}\left(1-\frac{r_{B}}{r}\right)}{1-\frac{r_{B}}{r} \frac{K^{c p}}{K^{p d}}},
$$

which follows from the decomposition of $\mathrm{CP}$ radar backscatter in Eq. (10), and where $r_{B}=\sigma_{0 B}^{c p} / \Delta \sigma_{0}$ and $r=\sigma_{0}^{c p} / \Delta \sigma_{0}$ are the clean sea surface CP-to-PD ratios for the Bragg scattering model and for the observed values listed in Table I.

Since the background (clean surface) PR corresponds to the observations, we also get an acceptable agreement between simulated and observed contrasts in the VV and HH NRCS (see Fig. 7 for the VV contrasts). Note that, unlike the model PD contrasts, the NP and CP contrasts have a clear trend with increasing film elasticity, $E_{f}$. As mentioned in section II, the Bragg NRCS should not be sensitive to the film type, unless $k_{B}$ gets into the interval between the cutoff wavenumbers for slicks of different types. The wave breaking contrasts, however, are related to the integral properties of the surface film damping, i.e., the larger the elasticity, $E_{f}$, the wider the spectral interval of the suppressed surface roughness (see Eq. (21) and Fig. 2). This can thus open for new opportunities in using NP and CP contrasts for slick type classification.

\section{CONCLUSION}

Radarsat-2 C-band quad-polarization SAR observations of crude oil, emulsion, and plant oil slicks acquired in the wind speed range from 4 to $8 \mathrm{~m} \mathrm{~s}^{-1}$, at incidence angles from $30^{\circ}$ to $50^{\circ}$ are analyzed in order to yield a deeper insight into the radar imaging mechanisms of surface slicks and their discrimination from look-alikes.

The co-polarized images are decomposed into radar scattering from regular (non-breaking) surface and wave breaking. For the dual co-polarized images, these components are (i) polarization difference (PD, $\Delta \sigma_{0}=\sigma_{0}^{v v}-\sigma_{0}^{h h}$ ) describing resonant Bragg scattering, and (ii) non-polarized (NP) contributions to VV and $\mathrm{HH}$ related to radar returns from breaking waves [16]. The impact of wave breaking on the CP NRCS is defined as a residual part of the total CP NRCS after subtraction of CP Bragg scattering, evaluated using the PD signal.

In line with previous studies [13], [14], we have found that wave breaking significantly contributes to both coand cross polarized NRCS at the background conditions (clean surface). NP radar returns from breaking waves 
here constitute about $20-40 \%$ (depending on the incidence angle) of the total VV NRCS, and about $60 \%$ of the HH NRCS. At cross-polarization, the observed contribution of wave breaking to the total CP NRCS varies from 50\% to $65 \%$.

Among all the considered radar scattering properties, the PD contrasts in the slicks have the largest values. Because the PD provide direct estimate of the damping of Bragg waves in slicks, this is not surprising. More surprisingly, however, it is found that the NP radar backscatter is clearly impacted by surface film, although the contrasts are much lower than in PD. The magnitudes of the CP contrasts are lower than predicted by the two-scale Bragg model (associated with measured PD contrasts), but comparable to the NP contrasts.

Since the suppression of Bragg waves in slicks is stronger than the suppression of wave breaking (associated with NP), the polarization ratio ( $\mathrm{PR}, \sigma_{0}^{h h} / \sigma_{0}^{v v}$ ), in slick areas is systematically higher than in the ambient areas, and that confirms the experimental findings reported in [16]. The PR in slicks approaches unity due to dominant NP radar backscatter. This can be used to discriminate between low-wind areas and slicks, which may look similar in the co- and cross-polarization images.

The contrasts of all the radar scattering properties demonstrate a clear trend to decrease with increasing wind speed. No clear dependence on the radar-look-directions have been revealed in any radar scattering properties. As observed, the crude oil and emulsion slicks cause stronger damping than plant oil.

The radar imaging model suggested in [15] has been revised in order to improve the description of wave breaking and to include the effect of sea surface film. It is suggested that this effect is related to a smoothing of the crests of incipient breakers by surfactants, reducing the crest instability growth rate, and thus, the energy flux generating intense surface crest roughness. The spectrum of this roughness is treated as a Kolmogorov-type spectrum. The diminution of both the instability rate and subsequent energy flux by the slicks, leads to decrease of the crest roughness parameters defining the model radar backscatter, i.e., the MSS and spectral level of the breaking crest roughness around the Bragg wavenumber, as well as the area covered by the individual breaking zones.

The suggested physical model is capable of explaining the observed modulations of both the NP and the CP radar backscatter. The model simulations show that, contrary to the resonant scattering, the reduction of the breaking intensity in slicks more strongly depends on the slick type. Depending on the background wind conditions, this opens for opportunities to consider NP and CP measurements for slick classification, and offers a consistent framework for future investigations of SAR signatures of sea surface slicks.

\section{ACKNOWLEDGMENT}

This work was supported through the EU FP7 project SeaU under contract no. 263246, the Russian Science Foundation Grant no. 15-17-20020, and the Ministry of Science and Education projects no. 5.2483.2014/K. The Radarsat-2 data used in the study was provided by NSC/KSAT under the Norwegian-Canadian Radarsat agreement 2008-2013. The data analysis was performed with the open-source Nansat python package [29].

\section{REFERENCES}

[1] H. Hühnerfuss and W. D. Garrett, "Experimental sea slicks: their practical applications and utilization for basic studies of air-sea interactions," J. Geophys. Res., vol. 86, pp. 439-447, 1981. 
[2] S. A. Ermakov, A. M. Zujkova, A. R. Panchenko, S. G. Salashin, T. G. Talipova, and V. I. Titov, "Surface film effect on short wind waves," Dynamics of Atmospheres and Oceans, vol. 10, pp. 31-50, Feb. 1986.

[3] S. A. Ermakov, S. G. Salashin, and A. R. Panchenko, "Film slicks on the sea surface and some mechanisms of their formation," Dynamics of Atmospheres and Oceans, vol. 16, pp. 279-304, Jan. 1992.

[4] W. Alpers and H. Hühnerfuss, "The damping of ocean waves by surface films: a new look at an old problem," Journal of Geophysical Research-Oceans, vol. 94, pp. 6251-6265, 1989.

[5] M. Gade, W. Alpers, H. Hühnerfuss, and P. A. Lange, "Wind wave tank measurements of wave damping and radar cross sections in the presence of monomolecular surface films," Journal of Geophysical Research, vol. 103, pp. 3167-3178, 1998.

[6] M. Gade, W. Alpers, H. Hühnerfuss, H. Masuko, and T. Kobayashi, "Imaging of biogenic and anthropogenic ocean surface films by the multifrequency/multipolarization SIR-C/X-SAR,” Journal of Geophysical Research, vol. 103, pp. 18 851-18 866, 1998.

[7] M. Gade, W. Alpers, H. Hühnerfuss, V. Wismann, and P. Lange, "On the reduction of the radar backscatter by oceanic surface films: Scatterometer measurements and their theoretical interpretation," Remote Sensing of Environment, vol. 66, no. 1, pp. 52-70, 1998.

[8] S. Skrunes, C. Brekke, T. Eltoft, and V. Kudryavtsev, "Comparing near coincident c- and x-band sar acquisitions of marine oil spills," IEEE Transactions on Geoscience and Remote Sensing, vol. 53, no. 4, 2015.

[9] H. A. Espedal, O. M. Johannessen, J. A. Johannessen, E. Dano, D. R. Lyzenga, and J. C. Knulst, "Coastwatch’95: Ers 1/2 sar detection of natural film on the ocean surface," Journal of Geophysical Research: Oceans, vol. 103, no. C11, pp. 24 969-24 982, 1998. [Online]. Available: http://dx.doi.org/10.1029/98JC01660

[10] B. Zhang, W. Perrie, X. Li, and W. G. Pichel, "Mapping sea surface oil slicks using radarsat-2 quad-polarization sar image," Geophysical Research Letters, vol. 38, p. 10602, May 2011.

[11] B. Minchew, "Determining the mixing of oil and sea water using polarimetric synthetic aperture radar," Geophysical Research Letters, vol. 39, AUG 232012.

[12] Junjun Yin, Jian Yang, Zheng-Shu Zhou, and Jianshe Song, "The Extended Bragg Scattering Model-Based Method for Ship and Oil-Spill Observation Using Compact Polarimetric SAR," IEEE Journal of Selected Topics in Applied Earth Observations and Remote Sensing, vol. 8, no. 8, pp. 3760-3772, August 2015 .

[13] V. N. Kudryavtsev, D. Hauser, G. Caudal, and B. Chapron, "A semiempirical model of the normalized radar cross-section of the sea surface 1. Background model," Journal of Geophysical Research (Oceans), vol. 108, pp. 8054-+, Jan. 2003.

[14] A. A. Mouche, D. Hauser, and V. Kudryavtsev, "Radar scattering of the ocean surface and sea-roughness properties: A combined analysis from dual-polarizations airborne radar observations and models in C band," Journal of Geophysical Research-Oceans, vol. 111, no. C9, SEP 12006.

[15] V. N. Kudryavtsev, D. Akimov, J. A. Johannessen, and B. Chapron, "On radar imaging of current features: 1. Model and comparison with observations," Journal of Geophysical Research-Oceans, vol. 110, no. C7, Jul. 2005.

[16] V. N. Kudryavtsev, B. Chapron, A. G. Myasoedov, F. Collard, and J. A. Johannessen, "On dual co-polarized sar measurements of the ocean surface," Geoscience and Remote Sensing Letters, IEEE, vol. 10, no. 4, pp. 761-765, July 2013.

[17] V. Kudryavtsev, I. Kozlov, B. Chapron, and J. A. Johannessen, "Quad-polarization sar features of ocean currents," J. Geophys. Res.-Oceans, vol. 119, 2014.

[18] D. Ivonin, S. Skrunes, C. Brekke, and A. Ivanov, "Interpreting sea surface slicks on the basis of the normalized radar cross-section model using RADARSAT-2 co-polarization dual-channel SAR images," Geophysical Research Letters, 2016.

[19] S. Skrunes, C. Brekke, and T. Eltoft, "Characterization of marine surface slicks by radarsat-2 multi-polarization features," IEEE Transactions on Geoscience and Remote Sensing, vol. 52, no. 9, pp. 5302-5319, September 2014.

[20] O. M. Phillips, The Dynamics of the Upper Ocean. Cambridge: Cambridge University Press, 1980.

[21] O. M. Phillips, "Spectral and Statistical Properties of the Equilibrium Range in Wind-Generated Gravity-Waves," Journal of Fluid Mechanics, vol. 156, no. JUL, pp. 505-531, 1985.

[22] T. Elfouhaily, D. Thompson, D. Vandemark, and B. Chapron, "A new bistatic model for electromagnetic scattering from perfectly conducting random surfaces," Waves in Random Media, vol. 9, no. 3, pp. 281-294, 1999. [Online]. Available: http://dx.doi.org/10.1088/0959-7174/9/3/301

[23] M. V. Yurovskaya, V. A. Dulov, B. Chapron, and V. N. Kudryavtsev, "Directional short wind wave spectra derived from the sea surface photography," Journal of Geophysical Research: Oceans, vol. 118, no. 9, pp. 4380-4394, 2013. [Online]. Available: http://dx.doi.org/10.1002/jgrc.20296 
[24] V. Kudryavtsev, A. Myasoedov, B. Chapron, J. A. Johannessen, and F. Collard, "Joint sun-glitter and radar imagery of surface slicks," Remote Sensing of Environment, vol. 120, no. SI, pp. 123-132, MAY 152012.

[25] A. Luscombe, "Image quality and calibration of radarsat-2," in Geoscience and Remote Sensing Symposium,2009 IEEE International,IGARSS 2009, vol. 2, July 2009, pp. II-757-II-760.

[26] E. A. Ericson, D. R. Lyzenga, and D. T. Walker, "Radar backscatter from stationary breaking waves," Journal of Geophysical ResearchOceans, vol. 104, no. C12, pp. 29679-29695, DEC 151999.

[27] V. Kudryavtsev, B. Chapron, and V. Makin, "Impact of wind waves on the air-sea fluxes: A coupled model," J. Geophys. Res.-Oceans, vol. 119, p. 1217-1236, 2014.

[28] M. Longuet-Higgins and R. Cleaver, "Crest instabilities of gravity waves. part 1. the almost highest wave." J. Fluid Mech., vol. 258, pp. 115-129, 1994.

[29] A. Korosov, M. W. Hansen, A. Yamakawa, K.-F. Dagestad, A. Vines, M. Riechert, A. Myasoedov, and E. A. Morozov, "nansat: Nansat v0.6.7 stable," Jan. 2016. [Online]. Available: http://dx.doi.org/10.5281/zenodo.45188 